\title{
Diversidade (alfa, beta e gama) da comunidade fitoplanctônica de quatro lagos artificiais urbanos do município de Goiânia, GO
}

\author{
Ina de Souza Nogueira ${ }^{1,3}$, João Carlos Nabout ${ }^{1,2}$, Juliano Eduardo Oliveira ${ }^{1}$ e Kamila Duarte Silva ${ }^{1}$
}

Recebido: 15.09.2006; aceito: 30.05 .2008

\begin{abstract}
Diversity (alpha, beta and gama) of phytoplankton community at four artificial lakes in Goiânia city, GO). The aim of this study was to compared the number of phytoplankton species (diversity alpha) in rainy and dry season at four urbane lakes in Goiânia city, as well was to value the total phytoplankton richness of each lake (diversity gama) and estimate this richness used extrapolator richness, and still to investigate the changes in phytoplankton compositions between the lakes (diversity beta). Finally, checked the influence of the abiotic variables on the biological index. During the study period they 325 species were registered. The four lakes presented different limnological characteristics and high species richness: Lake of Goiânia Botanical Garden (194 taxa), lake of Vaca Brava park (171), Rosas Lake (211) and lake of Bosque dos Buritis (179) and reduced floristic similarity. High beta diversity ( $\beta-1)$ was observed varying from 42 (February of 2001) to 64 (October of 2001), and reduced floristic similarity.
\end{abstract}

Key words: eutrophics lakes, species richness, floristic similarity

RESUMO - (Diversidade (alfa, beta e gama) da comunidade fitoplanctônica de quatro lagos artificiais urbanos do município de Goiânia, GO). O objetivo desse estudo foi comparar o número de espécies fitoplanctônicas (alfa diversidade) em períodos de chuva e seca de quatro lagos urbanos do município de Goiânia, bem como avaliar a riqueza total de espécies de cada lago (gama diversidade) e estimar essa riqueza usando extrapoladores de riqueza, e ainda investigar as mudanças nas composições fitoplanctônica entre os lagos (beta diversidade). Por fim, averiguar a influência das variáveis abióticas sobre os índices biológicos abordados. Durante o período de estudo um total de 325 espécies foram inventariadas. Os quatro lagos apresentaram características limnológicas distintas e apresentaram elevada riqueza de espécies: lago do Jardim Botânico (194 táxons), o lago do Parque Vaca Brava (171), Lago das Rosas (211) e o lago do Bosque dos Buritis (179). Observou-se baixa similaridade florística e elevada beta diversidade ( $\beta$-1) variando de 42 (fevereiro de 2001) a 64 (outubro de 2001). Palavras-chave: lagos eutróficos, riqueza de espécies, similaridade florística

\section{Introdução}

As estratégias para a conservação e conhecimento da biodiversidade são baseadas principalmente em composição florística e riquezas de espécies. Para Magurran (2004), diversidade alfa $(\alpha)$ ou diversidade local é o número total de espécies em um habitat, diversidade gama $(\gamma)$ ou diversidade regional é o número total de espécies observado em todos os habitats, e diversidade beta ( $\beta$ ) é a de mudança de espécies ao longo de um gradiente ambiental. Para Ward et al. (1999) a beta diversidade provê uma nova perspectiva para o conhecimento de áreas a serem preservadas. Para o Brasil, até o presente existem dois trabalhos que relatam o estudo da beta diversidade fitoplanctônica, Huszar et al. (1990) e Nabout et al. (2007) sendo esse último realizado em planícies de inundação.

Para Gaston (1996), o número de espécies amostradas em uma comunidade depende do "esforço amostral". Sendo que, quanto mais exaustivo for esse esforço mais próximo o número de espécies amostradas estará do valor real da comunidade. Porém o esforço amostral depende fundamentalmente de auxilio financeiro, disponibilidade de tempo e acessos a diferentes ambientes. No entanto, as dificuldades para conhecer o número de real de espécies são solucionadas com o auxilio de ferramentas estatísticas permitindo estimar o número de espécies por extrapolação (Lee \& Chao 1994). Dessa forma, os extrapoladores de

1. Universidade Federal de Goiás, ICB I, Departamento de Biologia Geral, Laboratório de Limnologia. Caixa Postal 131, 74001-970 Goiânia, GO, Brasil

2. Programa de Doutorado em Ciências Ambientais, Universidade Federal de Goiás. Caixa Postal 131, 74001-970 Goiânia, GO, Brasil

3. Autor para correspondência: nogueira@icb.ufg.br 
riqueza constituem uma ferramenta para respaldar inventários fornecendo aos pesquisadores a estimativa de suficiência ou não do trabalho executado.

Os estudos de fitoplâncton de lagos urbanos vêem assumindo grande importância, principalmente devido esses lagos propiciarem benefícios promovidos pela qualidade cênica à população urbana. No Brasil exitem alguns trabalhos sobre fitoplâncton de ecossistemas urbanos, alguns deles desenvolvidos até o ano de 1999 encontram-se citados em Nogueira \& LeandroRodrigues (1999), destacam-se ainda os trabalhos de Menezes (1999), Giani et al. (1999), Silva (1999), Ferreira \& Menezes (2000), Tucci et al. (2006). A cidade de Goiânia é muito rica em corpos d'água artificiais distribuídos entre os inúmeros parques que são destinados a áreas de lazer da população ou de preservação ambiental, no entanto o estudo da comunidade fitoplanctônica nesses lagos ainda é insipiente. Somente de Nogueira \& LeandroRodrigues (1999), abordou o fitoplâncton desses lagos, no caso o lago do Jardim Botânico Chico Mendes.

O presente estudo teve como objetivo comparar o número de espécies fitoplanctônicas (diversidade alfa) em períodos de chuva e seca de quatro lagos urbanos do município de Goiânia, bem como avaliar a riqueza total de espécies de cada lago (diversidade gama) e estimar essa riqueza usando extrapoladores de riqueza, e ainda investigar as mudanças nas composições fitoplanctônica entre os lagos (diversidade beta). Por fim, averiguar a influência das variáveis abióticas sobre os índices biológicos abordados.

\section{Material e métodos}

Os lagos rasos artificiais estudados estão situados em áreas de lazer do município de Goiânia, Goiás: lago do Jardim Botânico de Goiânia (16 43'347'S e $\left.49^{\circ} 15^{\prime} 120^{\prime} \mathrm{W}\right)$, lago do Parque Vaca Brava $\left(16^{\circ} 42^{\prime} 50^{\prime \prime} \mathrm{S}\right.$ e $\left.49^{\circ} 16^{\prime} 250^{\prime \prime} \mathrm{W}\right)$, lago das Rosas (16 40'76"S e 49 16'430"W) e o lago do Bosque dos Buritis $\left(16^{\circ} 40^{\prime} 285^{\prime}\right.$ 'S e $\left.49^{\circ} 15^{\prime} 618^{\prime} \mathrm{W}\right)$. As variáveis morfométricas dos quatros lagos foram efetuadas a partir de medidas de perímetro, comprimento máximo, largura máxima e profundidade média, além disso, observações em campo das características paisagísticas encontram-se relatadas nesse trabalho.

O Estado de Goiás caracteriza-se por ter um clima quente, variando de úmido a semi-árido, com até cinco meses de seca (Galinkin 2002). Segundo a classificação de Köppen, enquadra-se no tipo Aw, característico dos climas úmidos tropicais, com duas estações bem definidas: seca, no inverno, e úmida, no verão. Os dados de precipitação foram adquiridos em estações metereológicas o mais próximo possível do local de estudo, disponibilizadas pela Secretaria de Ciência e Tecnologia do Estado de Goiás (Sectec-GO).

As amostragens foram realizadas na subsuperfície da água de cada lago nos meses agosto e setembro de 2000 (meses de seca), fevereiro e março (meses de chuva), junho e agosto (meses de seca), outubro e novembro de 2001 (meses de chuva).

Para estimar a riqueza total de espécies foram consideradas tanto amostras destinadas às análises quantitativas quanto qualitativas. Para a análise quantitativa do fitoplâncton foram coletadas amostras em frascos de $100 \mathrm{ml}$ e de um litro, sendo fixadas com lugol-acético (Vollenweider 1974) e as qualitativas fixadas com solução Transeau (Bicudo \& Menezes 2006) e foram depositadas no herbário da Universidade Federal de Goiás (UFG 26788 a 26823; 26895 a 26906; 26957 a 26960). A densidade do fitoplâncton foi estimada pelo método de Utermöhl (1958) em microscópio invertido Zeiss (modelo Axiovert 25) de 450 vezes, utilizando-se tempo de sedimentação de pelo menos três horas para cada centímetro de altura da câmara (Margalef 1983). A diversidade específica foi calculada usando o índice de Shannon-Wiener (Shannon \& Weaver 1963). O sistema de classificação adotado para as classes taxonômicas foi o de Van den Hoeck et al. (1993) e para níveis genéricos e infragenéricos foram Komárek \& Anagnostidis (1989 e 1999) para Cyanobacteria, Komárek \& Fott (1983), Comas (1996), Hindák (1990) para Chlorophyceae, Prescott \& Bicudo (1981) e Prescott et al. (1982), para Desmidiales, Etll (1983) para Tribophyceae, Starmach (1985) para Chrysophyceae, e Krammer \& Lange-Bertalot (1991) para Bacillariophyceae.

As variáveis abióticas analisadas foram: temperatura da água, $\mathrm{pH}$ e saturação de oxigênio utilizando o multianalisador de água Hach DR2000, condutividade elétrica (condutivímetro portátil Hach 44600), cor (método espectrofotométrico), turbidez (turbidímetro Hach 2100P), demanda bioquímica de oxigênio, demanda química de oxigênio e ortofosfato (Clesceri et al. 1992), dureza total e cloreto (APHA 1995). A transparência da água foi medida pelo disco de Secchi e a profundidade aferida por meio de uma trena.

A análise de variância (ANOVA fator único; nível de significância de 0,05 ) foi utilizada para os dados de densidade e diversidade fitoplanctônica a fim de detectar alguma diferença entre os lagos amostrados. 
A análise de componentes principais (ACP) foi feita com a matriz de correlação entre as variáveis abióticas, sendo que as variáveis utilizadas foram: profundidade, transparência, saturação de oxigênio, pH, condutividade, ortofosfato e turbidez. Previamente, a transformação logarítmica $(\log (n+1))$ dos dados foi aplicada em todas variáveis, exceto pH. A análise foi efetuada pelo programa PC-ORD (McCune \& Mefford 1997).

A riqueza de espécies foi baseada em dados de incidência (presença/ausência) (Chazdon et al. 1998). Os estimadores de riqueza de espécies usados para esse estudo foram o Jacknife de primeira e segunda ordem (Sjack1 e Sjack2, respectivamente) (Burnham \& Overton 1978) e Schao2 (Chao 1987). Todos foram calculados utilizando o programa EstimateS (Colwell 1997).

$\mathrm{O}$ índice de diversidade beta utilizado foi o $\beta$-1 (Harrison et al. 1992), o qual mede o quanto a diversidade regional excede a diversidade alfa média. Afórmula para esse índice é: $\beta-1=\left[\left(\mathrm{S} / \alpha_{\text {medio }}\right)-1\right] /[\mathrm{N}-1]$ $\mathrm{x} 100$, no qual, $\mathrm{S}$ é diversidade regional ou riqueza total (número de espécies do grupo de lagos de cada período), $\alpha_{\text {médio }}$ é diversidade alfa média (número médio de espécies) para o grupo de lagos de cada período, $\mathrm{N}$ é número de lagos do período.

A similaridade florística entre os lagos foi medida pela análise de agrupamento aplicado para a distância de índice de Jaccard (Jaccard 1908) utilizando-se o método de ligação UPGMA (Unweight Pair-Group Method Average) (Sneath \& Sokal 1973), sendo avaliado o coeficiente de correlação cofenético (r). Todas essas análises foram realizadas pelo programa NTSYSpc, versão 2.0.

\section{Resultados e Discussão}

As amostragens foram realizadas nos períodos de seca e chuva, sendo que o mês de novembro de 2001 apresentou o maior período de precipitação, enquanto que os menores ocorreram em junho e agosto (figura 1). Os quatro reservatórios estão inseridos na região metropolitana de Goiânia, e apresentam diferentes características morfométricas (tabela 1) e limnológicas (tabela 2), alguns com elevada transparência (Lago do Jardim Botânico), outros com reduzida transparência e elevada turbidez (Lago do Parque Vaca Brava e Lago das Rosas) e outros ainda com elevados valores de saturação de oxigênio (Lago do Bosque dos Buritis) (tabela 2). Porém de forma geral, os quatro lagos tenderam a eutróficos, principalmente devido aos altos valores de ortofosfato e elevada densidade fitoplanctônica registradas em diversos períodos amostrais (tabelas 2, 3). ANOVA revelou que os lagos apresentaram uma densidade fitoplanctônica significativamente diferente $(\mathrm{F}=$ 4,009; $p=0,02 ; \mathrm{n}=4$ ) e a diversidade de espécies também foi significativamente diferente $(\mathrm{F}=4,74 ; p$ $=0,009 ; \mathrm{n}=4)$.

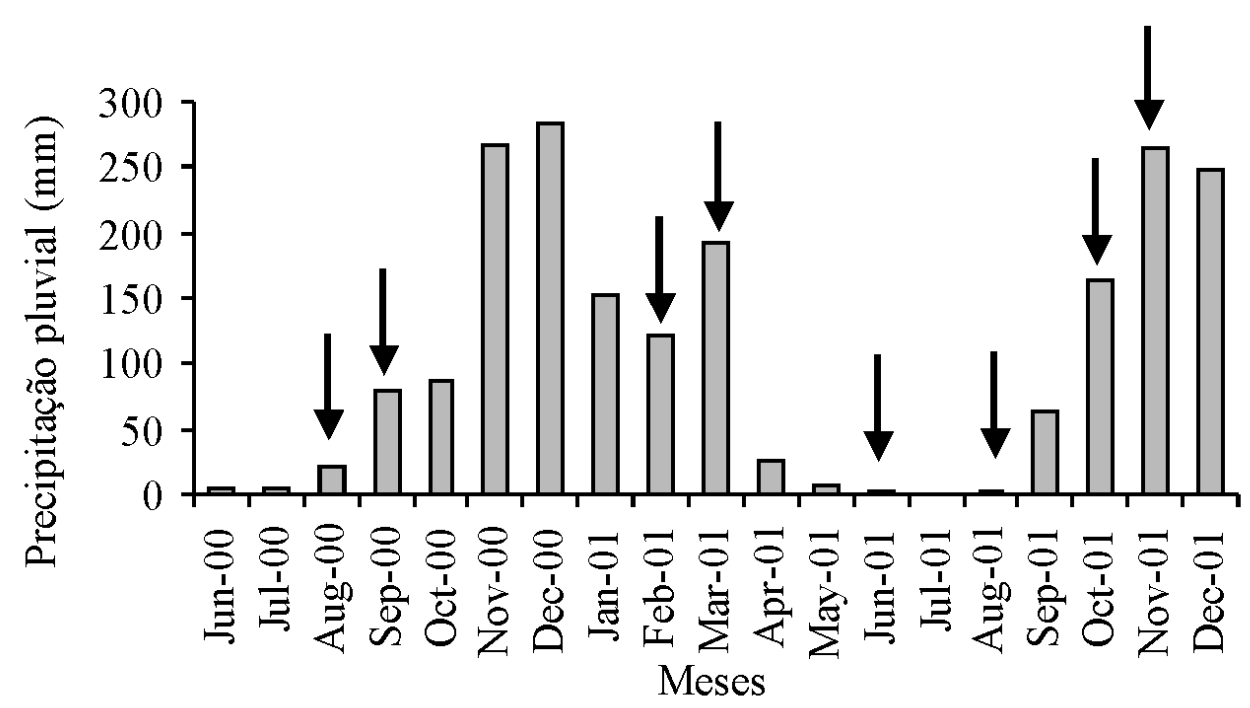

Figura 1. Totais pluviométricos mensais dos anos de 2000 e 2001 em Goiânia. As setas indicam os períodos de coleta. 
Tabela 1. Dados morfométricos dos quatro lagos urbanos estudados em Goiânia.

\begin{tabular}{|c|c|c|c|c|}
\hline & $\begin{array}{l}\text { Lago do Jardim } \\
\text { Botânico }\end{array}$ & $\begin{array}{c}\text { Lago do Parque } \\
\text { Vaca Brava }\end{array}$ & Lago das Rosas & $\begin{array}{c}\text { Lago do Bosque dos } \\
\text { Buritis }\end{array}$ \\
\hline Profundidade média (m) & 2,11 & 1,53 & 0,83 & 1,41 \\
\hline Comprimento máximo (m) & 378,34 & 171,11 & 192,88 & 123,26 \\
\hline Largura máxima (m) & 60,94 & 92,91 & 96,9 & 91,13 \\
\hline Perímetro (m) & 918,43 & 487,24 & 522,75 & 327,82 \\
\hline
\end{tabular}

Observou-se através dos escores derivados da ACP que os lagos apresentaram-se limnologicamente distintos (figura 2). Os dois primeiros componentes principais explicaram $49,45 \%$ da variância total. A profundidade, transparência, condutividade, saturação de oxigênio e turbidez correlacionaram-se positivamente com o primeiro componente principal, enquanto que a saturação de oxigênio correlacionou-se positivamente com o segundo componente principal (tabela 4). Essas variáveis abióticas foram de fundamental importância

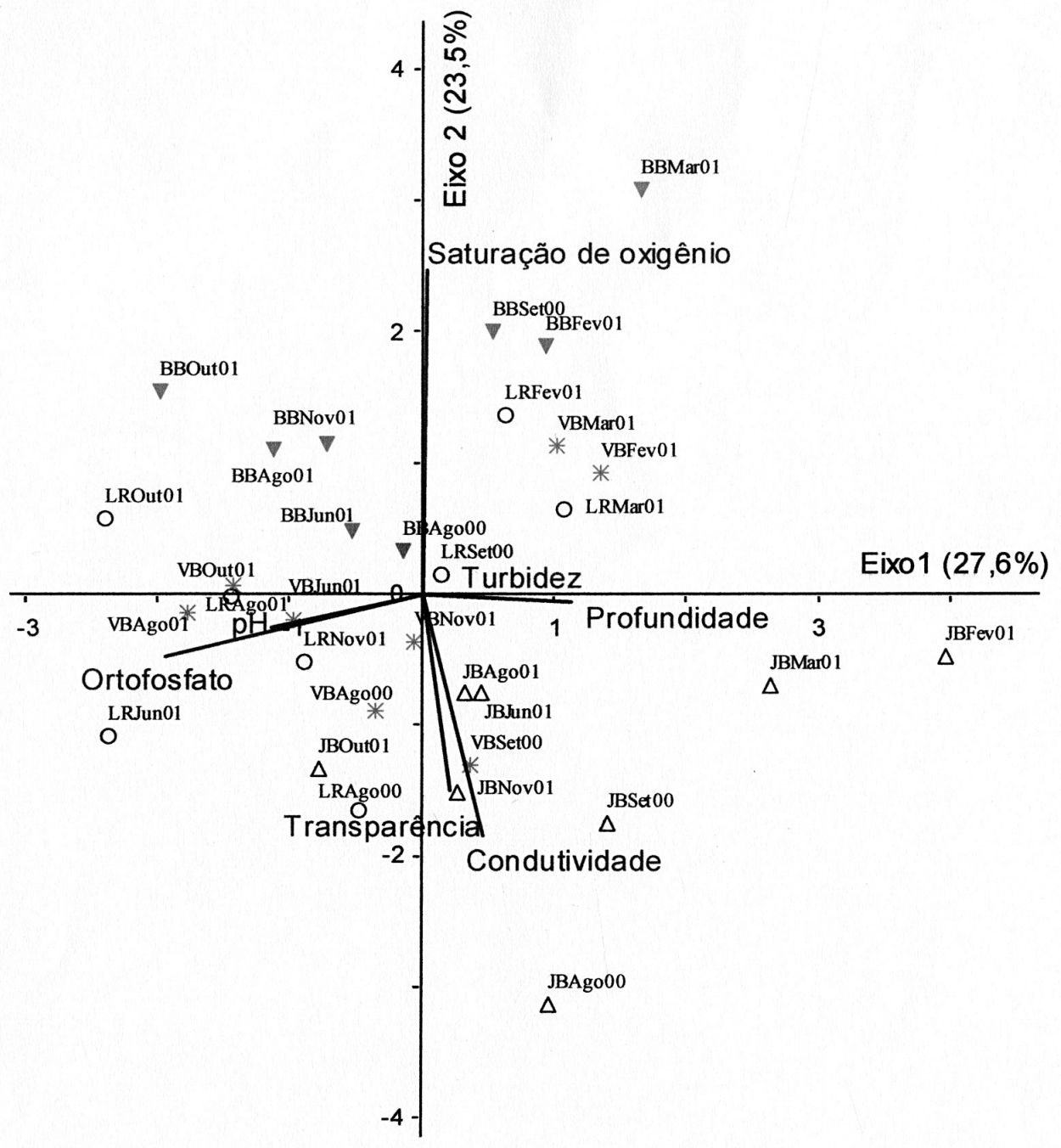

Figura 2. Escores derivados da ACP para dados ambientais. Lagos: $(\triangle)$ Lago do Jardim Botânico; (O) Lago das Rosas; $(*)$ Lago do Parque Vaca Brava; $(\boldsymbol{\nabla})$ Lago do Bosque dos Buritis. Meses: Ago = Agosto; Set = Setembro; Out = Outubro; Nov = Novembro; Fev = Fevereiro; Mar = Março e Jun = Junho. Anos: $00=2000$ e $01=2001$. 
Tabela 2. Valores médios e desvio padrão (entre parênteses) de variáveis limnológicas encontradas nos quatro lagos estudados (n=8).

\begin{tabular}{lrrrr}
\hline & $\begin{array}{c}\text { Lago do Jardim } \\
\text { Botânico }\end{array}$ & $\begin{array}{c}\text { Lago do Parque } \\
\text { Vaca Brava }\end{array}$ & Lago das Rosas & \multicolumn{1}{c}{$\begin{array}{c}\text { Lago do Bosque } \\
\text { dos Buritis }\end{array}$} \\
\hline Transparência (m) & $0,92(0,09)$ & $0,51(0,88)$ & $0,50(0,08)$ & $0,48(0,12)$ \\
Temperatura da água $\left({ }^{\circ} \mathrm{C}\right)$ & $24,68(2,03)$ & $26,07(1,94)$ & $26,87(2,53)$ & $26,78(3,94)$ \\
Dureza Total $\left(\mathrm{mg} \mathrm{L}^{-1}\right)$ & $61(14,06)$ & $64(13,18)$ & $57,12(17,19)$ & $51,87(14,14)$ \\
Condutividade $\left(\mu \mathrm{s} \mathrm{cm}^{-1}\right)$ & $133,41(13,67)$ & $114,82(16,34)$ & $125,98(24,52)$ & $95,23(14,08)$ \\
pH & $7,13(0,42)$ & $7,51(1,29)$ & $7,43(1,36)$ & $6,92(0,74)$ \\
DBO5 a 20 ${ }^{\circ} \mathrm{C}\left(\mathrm{mg} \mathrm{L}^{-1}\right)$ & $2,51(1,01)$ & $2,47(1,37)$ & $2,82(1,43)$ & $3,26(1,94)$ \\
DQO $\left(\mathrm{mg} \mathrm{L}^{-1}\right)$ & $19,62(11,85)$ & $23,75(14,18)$ & $25,75(11,69)$ & $33,73(14,11)$ \\
Saturação de Oxigênio $(\%)$ & $83,06(21,54)$ & $83,43(21,54)$ & $87,18(23,7)$ & $118,12(64,14)$ \\
Ortofosfato $(\mu \mathrm{g} \mathrm{L})$ & $55(50,43)$ & $83,75(54,23)$ & $83,75(54,75)$ & $80(64,14)$ \\
Cloreto $\left(\mathrm{mg} \mathrm{L}^{-1}\right)$ & $8,87(1,48)$ & $2,56(0,77)$ & $10,31(13,4)$ & $6,31(2,67)$ \\
Cor (mg L $\left.{ }^{-1}\right)$ & $89,57(25,47)$ & $275,85(362,37)$ & $195,42(114,82)$ & $160,78(99,03)$ \\
Turbidez $(\mathrm{UNT})$ & $15,71(4,54)$ & $28,42(19,35)$ & $31,64(19,87)$ & $23,28(10,93)$ \\
\hline
\end{tabular}

para a separação dos lagos no espaço bivariado da ACP. O ortofosfato correlacionou-se negativamente com o primeiro componente principal, os lagos correlacionados com essa variável, principalmente o Lago da Vaca Brava e o Lago das Rosas na estação de seca e início de chuva (outubro). Além disso, o lago do Bosque dos Buritis em junho (estação seca) apresentou as maiores densidades do fitoplâncton e, também foi correlacionado com o ortofosfato. O ortofosfato é uma forma de fósforo e importante reguladora do crescimento do fitoplâncton (Scheffer 1999).

O Lago do Jardim Botânico foi o lago artificial com maior profundidade (período chuvoso) e elevada transparência (período de estiagem); o Lago do Parque Vaca Brava e o Lago das Rosas apresentaram-se limnologicamente semelhantes, com elevados valores

Tabela 3. Densidade fitoplanctônica (ind $\mathrm{ml}^{-1}$ ) e diversidade específica (bits ind ${ }^{-1}$ ) (entre parênteses) registrada em cada lago durante todo o período de estudo. Resumo estatístico e ANOVA (nível de significância 0,05) entre os lagos.

\begin{tabular}{lccrc}
\hline & $\begin{array}{c}\text { Lago do Jardim } \\
\text { Botânico }\end{array}$ & $\begin{array}{c}\text { Lago do Parque } \\
\text { Vaca Brava }\end{array}$ & Lago das Rosas & \multicolumn{2}{c}{$\begin{array}{c}\text { Lago do Bosque } \\
\text { dos Buritis }\end{array}$} \\
\hline Ago/00 & não consta & não consta & não consta & não consta \\
Set/00 & $24.244(4,29)$ & $17.494(3,46)$ & $24.819(3,41)$ & $45.065(2,13)$ \\
Fev/01 & $7.454(4,05)$ & $50.839(1,12)$ & $7.199(3,89)$ & $40.005(2,41)$ \\
Mar/01 & $9.809(3,76)$ & $50.413(1,12)$ & $7.676(3,78)$ & $80.219(0,97)$ \\
Jun/01 & $42.358(2,04)$ & $80.914(2,81)$ & $45.805(3,41)$ & $118.648(3,63)$ \\
Ago/01 & $51.516(3,42)$ & $83.725(2,83)$ & $51.879(2,79)$ & $195.209(2,38)$ \\
Out/01 & $35.222(4,11)$ & $129.617(3,33)$ & $7.5519(3,93)$ & $59.663(3,54)$ \\
Nov/01 & $33.328(4,15)$ & $65.206(2,29)$ & $40.482(3,43)$ & $12.880(1,62)$ \\
Média & $28.433(3,69)$ & $68.833(2,42)$ & $36.925(0,47)$ & $89.801(2,38)$ \\
Desvio Padrão & $17.761(0,78)$ & $38.369(0,96)$ & $105.519(3,93)$ & $59.032(0,96)$ \\
Máximo & $51.516(4,29)$ & $129.617(3,46)$ & $7.199(2,79)$ & $195.209(3,63)$ \\
Mínimo & $7.454(2,04)$ & $17.494(1,12)$ & $40.005(0,971)$ \\
\hline F & & $4.009(4,74)$ & & \\
$p$ & & & &
\end{tabular}


Tabela 4. Correlação das variáveis limnológicas obtidas nos lagos de Goiânia com os dois primeiros eixos da ACP.

\begin{tabular}{lcc}
\hline Variáveis & Eixo 1 & Eixo 2 \\
\hline Profundidade & 0,4647 & $-0,089$ \\
Transparência & 0,1993 & $-0,4766$ \\
Condutividade elétrica & 0,2945 & $-0,5281$ \\
pH & $-0,4707$ & $-0,1926$ \\
Saturação de Oxigênio & 0,0658 & 0,6137 \\
Ortofosfato & $-0,6122$ & $-0,2685$ \\
Turbidez & 0,2385 & $-0,0147$ \\
\hline
\end{tabular}

de ortofosfato e $\mathrm{pH}$ (período de estiagem) e elevados valores de cor e turbidez (período chuvoso); o Lago do Bosque dos Buritis registrou elevados valores de temperatura da água e saturação de oxigênio. Os elevados valores de saturação de oxigênio encontrados nesse lago (tabela 2) devem-se à movimentação da água (provocado pelo chafariz) e, também, pela elevada densidade do fitoplâncton, particularmente as cianobactérias. Xiangacan (2003) registrou, na China, lagos urbanos eutrofizados com características semelhantes às registradas nos lagos urbanos de Goiânia, isto é, como baixa transparência, elevados valores de oxigênio dissolvido em decorrência do excesso de algas.

Durante todo o período de estudo, a riqueza total de espécies encontradas nos quatro lagos foi de 325 táxons. Destes, 216 táxons foram identificados em nível infragenérico (tabela 5). As espécies mais freqüentes foram: Peridiniopsis thompsonii, Coelastrum reticulatum, Dictyosphaeriumpulchellum, Monoraphidium contortum, Pediastrum duplex, Pediastrum simplex, Scenedesmus ellipticus, Desmodesmus maximus e Desmodesmus spinosus. Essas espécies foram registradas em todos os lagos estudados.

Dos quatro lagos estudados, o Lago das Rosas apresentou a maior riqueza total de espécies (diversidade gama) com 211 de táxons, enquanto o Lago do Parque Vaca Brava registrou a menor gama diversidade 171 táxons (figura 3). Em todos os lagos, a classe Chlorophyceae registrou a maior riqueza. A tabela 6 mostra os valores dos estimadores (Sjack1, Sjack2, e Schao2) para cada lago, sendo que o estimador não paramétrico Sjack1 evidenciou que a riqueza de espécies esperada no lago do Jardim Botânico foi amostrada em 70\%, no Lago do Parque Vaca Brava 68\%, no Lago das Rosas $72 \%$ e no Lago do Bosque dos Buritis 68\%.

A amostragem efetuada nos lagos analisados foi bastante representativa para o estudo florístico. Para o fitoplâncton somente o trabalho de Nabout et al. (2007) aborda o uso de extrapoladores de riqueza em lagoas de inundação do rio Araguaia, no entanto, outras áreas da limnologia têm usado desse recurso estatístico, tais como trabalhos com macrófitas aquáticas (Bini et al. 2001) e macroinvertebrados bentônicos (Melo \& Froehlich 2001, Melo et al. 2003) para reforçar a expressividade da amostragem.

A riqueza de espécies (diversidade alfa) apresentou oscilação temporal em todos os lagos estudados (figura

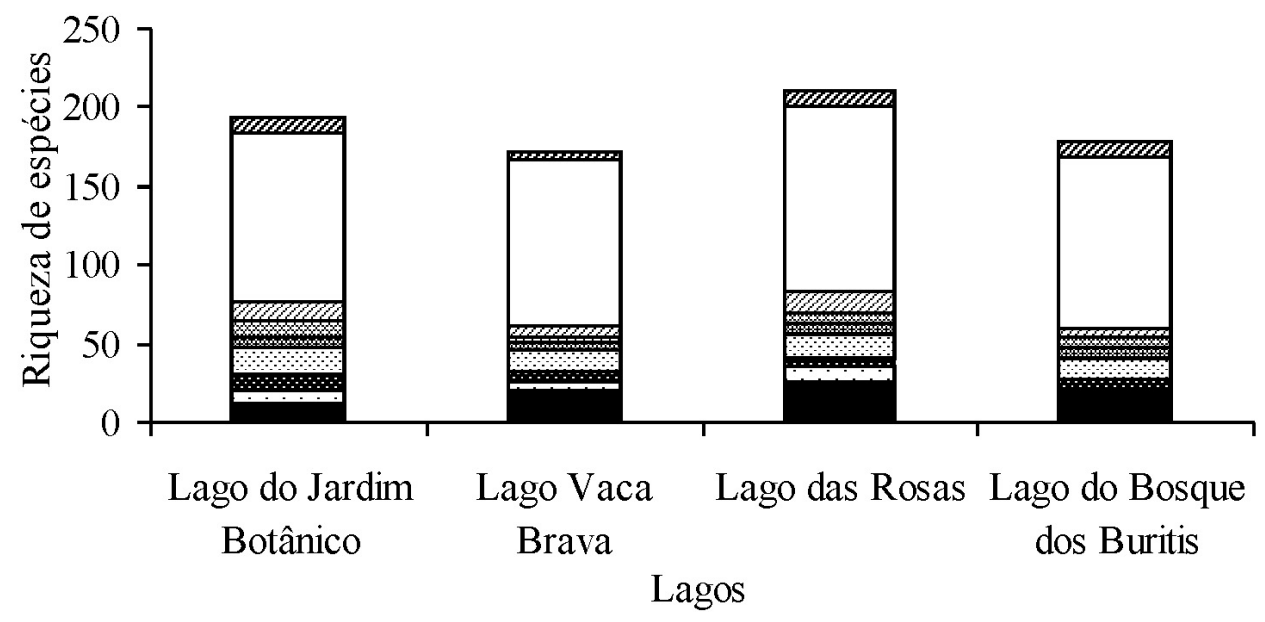

Figura 3. Número de espécies por lago durante todo o período de estudo (diversidade gama), distribuídos em classes taxonômicas presentes

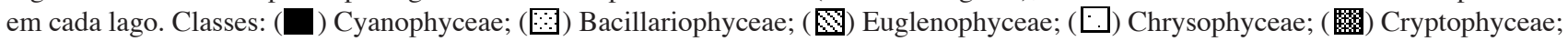

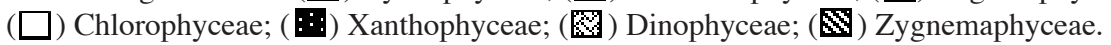




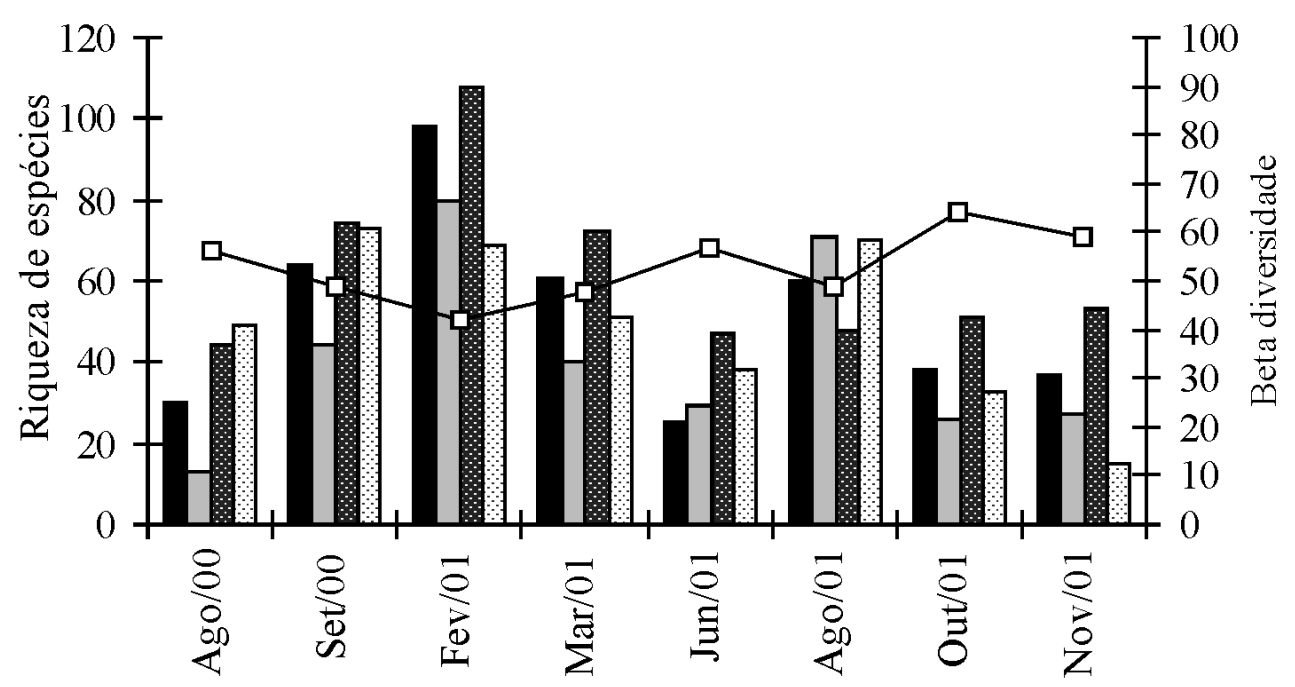

Períodos amostrais

Figura 4. Riqueza especifica (diversidade alfa) e diversidade beta ( $\square-$ ) durante todo o período amostral. Lagos: nico; (—) Vaca Brava; (1.: Lago das Rosas ( $)$ Bosque dos Buritis.

4), sendo que em fevereiro (período de chuva) os quatro lagos registraram a maior riqueza. A menor riqueza foi observada no Lago do Jardim Botânico, no Lago do Parque Vaca Brava e no Lago das Rosas em agosto de 2000 (período de estiagem). Enquanto o Lago do Bosque dos Buritis registrou a menor riqueza em novembro de 2001 (período de chuva).

No presente estudo, os lagos apresentaram riqueza específica total semelhante a de outros lagos eutróficos brasileiros. O Lago Paranoá (Branco \& Senna 1996; 76 táxons), Lagoa Pedreira (Talamoni \& Okano 1997; 87 táxons), Lagoa Douradas (Talamoni \& Okano 1997; 127 táxons) e Lago Monte Alegre (Silva 1999; 119 táxons) apresentaram riqueza inferior aos dos lagos urbanos de Goiânia. Assim como em outros lagos eutróficos (Talamoni \& Okano 1997, Silva 1999, Sant'Anna et al. 1989, Sant'Anna et al. 1997), a classe Chlorophyceae apresentou o maior número de táxons, o que também foi observado nos quatro lagos de Goiânia.

O índice de diversidade beta foi aplicado objetivando quantificar a renovação ou substituição de espécies entre os ambientes. $\mathrm{O}$ índice de diversidade beta $\beta$-1 para o período de estudo manteve-se acima de 40. O mês de outubro de 2001 registrou a maior $\beta-1$, neste mês os quatro lagos apresentaram um fitoplâncton mais distinto (figura 4). Huszar et al. (1990) utilizando a diversidade beta, observou que as lagoas do baixo rio Doce (Estado do Espírito Santo) apresentavam elevada diversidade beta, tendo apresentado uma elevada heterogeneidade ambiental. De forma semelhante, Nabout et al. (2007) observaram uma elevada diversidade beta fitoplanctônica nas lagoas de inundação do rio Araguaia.

O índice $\beta$-1 correlacionou-se positivamente com a densidade $(r=0,41 ; p=0,003 ; \mathrm{n}=8)$, dessa forma, os períodos amostrais que apresentaram elevadas densidades registraram também maiores valores de $\beta-1$. Os períodos de estiagem, como nos meses de março de 2001, junho de 2001, agosto de 2001 e outubro de 2001, os lagos apresentaram as maiores densidades e os menores valores de diversidade específica. Nestes meses também foram registradas ocorrências de florações de Cyanobacteria no Lago do Bosque dos Buritis e Lago do Parque Vaca Brava, de Chrysophyceae no Lago do Jardim Botânico e de Chlorophyceae no Lago das Rosas (Nabout \& Nogueira, dados não publicados). As condições ambientais que propiciaram as elevadas densidades nos quatro lagos favoreceram a ocorrência de uma comunidade fitoplanctônica distinta, elevando a beta diversidade e, em alguns períodos (chuvosos) a diversidade específica (tabela 3). Segundo Margalef (1972) a diversidade específica acima de 2,5 bits ind ${ }^{-1}$ é um valor elevado. 
Tabela 5. Lista dos táxons identificados em nível infragenérico e as localidades de ocorrência: JB = Lago do Jardim Botânico; VB = Lago do Parque Vaca Brava; LR = Lago das Rosas; BB = Lago do Bosque dos Buritis.

\begin{tabular}{|c|c|c|c|c|}
\hline Família / Espécie & JB & VB & LR & $\mathrm{BB}$ \\
\hline \multicolumn{5}{|l|}{ CYANOBACTERIA } \\
\hline Arthrospira jennerii Stizemb ex Gom. & & & $\mathrm{X}$ & \\
\hline Chroococcus limneticus Lemm. & & $\mathrm{X}$ & $\mathrm{X}$ & $\mathrm{X}$ \\
\hline Chroococcus minor (Kütz.) Näg. & $\mathrm{X}$ & $\mathrm{X}$ & $\mathrm{X}$ & $\mathrm{X}$ \\
\hline Chroococcus obliteratus P. G. Richt. & $\mathrm{X}$ & $\mathrm{X}$ & $\mathrm{X}$ & $\mathrm{X}$ \\
\hline Coelosphaerium kuetzingianum Näg. & & & $\mathrm{X}$ & $\mathrm{X}$ \\
\hline Gleiterinema amphibium (Ag. ex Gom.) Anagn. \& Kom. & $\mathrm{X}$ & $\mathrm{X}$ & $\mathrm{X}$ & $\mathrm{X}$ \\
\hline Gloeothece membranacea (Rabenh.) Bornet & & $\mathrm{X}$ & $\mathrm{X}$ & \\
\hline Jaaginema angustissimum (West \& G.S. West) Anagn. \& Kom. & & $\mathrm{X}$ & $\mathrm{X}$ & \\
\hline Jaaginema geminatum (Menegh. ex Gom.) Anagn. \& Kom. & & $\mathrm{X}$ & $\mathrm{X}$ & \\
\hline Merismopedia tenuissima Lemm. & $\mathrm{X}$ & $\mathrm{X}$ & $\mathrm{X}$ & \\
\hline Microcystis aeruginosa (Kütz.) Kütz. & $\mathrm{X}$ & $\mathrm{X}$ & $\mathrm{X}$ & $\mathrm{X}$ \\
\hline Microcystis cf. ichthyoblabe Kütz. & & $\mathrm{X}$ & $\mathrm{X}$ & $\mathrm{X}$ \\
\hline Microcystis protocystis Crow & & & $\mathrm{X}$ & \\
\hline Microcystis stagnalis Lemm. var. pulchra Lemm. & & $\mathrm{X}$ & $\mathrm{X}$ & $\mathrm{X}$ \\
\hline Microcystis wesenberghii (Kom.) Kom. in Kond. & & $\mathrm{X}$ & $\mathrm{X}$ & $\mathrm{X}$ \\
\hline Planktolyngbya circuncreta (G.S.West) Anagn. \& Kom. & & $\mathrm{X}$ & $\mathrm{X}$ & $\mathrm{X}$ \\
\hline Planktolyngbya limnetica (Lemm.) Kom.-Legn. \& Cronb. & $\mathrm{X}$ & $\mathrm{X}$ & $\mathrm{X}$ & $\mathrm{X}$ \\
\hline Radiocystis fernandoi Kom. \& Kom.-Legn. & & & $\mathrm{X}$ & $\mathrm{X}$ \\
\hline Spirulina gignatea Schmidle & $\mathrm{X}$ & $\mathrm{X}$ & & \\
\hline Synechococcus capitatus Bai.-Wat. \& Kom. & & & $\mathrm{X}$ & $\mathrm{X}$ \\
\hline Synechocystis aquatilis Sauvag. & $\mathrm{X}$ & $\mathrm{X}$ & $\mathrm{X}$ & $\mathrm{X}$ \\
\hline \multicolumn{5}{|l|}{ CHRYSOPHYCEAE } \\
\hline Chromulina $\mathrm{cf}$. mediostigmata Ettl & $\mathrm{X}$ & & & \\
\hline Chromulina cf. pyrenoidosa Ettl & $\mathrm{X}$ & & & \\
\hline Chrysococcus elipsoides Ettl & & & $\mathrm{X}$ & \\
\hline Chrysococcus rufescens Klebs & $\mathrm{X}$ & & $\mathrm{X}$ & \\
\hline Chysococcus punctiformis Pasch. & $\mathrm{X}$ & & & \\
\hline Dinobryon divergens Imhof & $\mathrm{X}$ & $\mathrm{X}$ & $\mathrm{X}$ & $\mathrm{X}$ \\
\hline Dinobryon sertularia Ehr. & $\mathrm{X}$ & $\mathrm{X}$ & $\mathrm{X}$ & \\
\hline Kephyrion cf. valkanovii Hub.-Pest. & $\mathrm{X}$ & & & \\
\hline Pseudokephyrion tatricum (Juris) Starm. & $\mathrm{X}$ & $\mathrm{X}$ & $\mathrm{X}$ & $\mathrm{X}$ \\
\hline \multicolumn{5}{|l|}{ TRIBOPHYCEAE } \\
\hline Centritractus belenophorus Lemm. & $\mathrm{X}$ & & & \\
\hline Ellipsoidion perminimum Pasch. & $\mathrm{X}$ & & & $\mathrm{X}$ \\
\hline Ellipsoidion stichococcoides Pasch. & $\mathrm{X}$ & & & $\mathrm{X}$ \\
\hline Goniochloris fallax Fott & $\mathrm{X}$ & $\mathrm{X}$ & $\mathrm{X}$ & \\
\hline Goniochloris mutica (A. Br.) Fott & & & & $\mathrm{X}$ \\
\hline Goniochloris sculpta Geitler & & & & $\mathrm{X}$ \\
\hline Pseudostaurastrum enorme (Ralfs) Chodat & & $\mathrm{X}$ & & \\
\hline Tetraedriella jovetii (Bourr.) Bourr. & & $\mathrm{X}$ & & \\
\hline Tetraedriella polychloris Skuja & $\mathrm{X}$ & & & \\
\hline Tetraedriella regularis (Kütz.) Fott & $\mathrm{X}$ & $\mathrm{X}$ & $\mathrm{X}$ & $\mathrm{X}$ \\
\hline Tetraedriella spinigera Skuja & $\mathrm{X}$ & & & $\mathrm{X}$ \\
\hline Tetraplektron torsum (Skuja) Dedos.-Sceg. & $\mathrm{X}$ & & & \\
\hline \multicolumn{5}{|l|}{ BACILLARIOPHYCEAE } \\
\hline Achnanthidium minutissimum (Kütz.) Czarn. & $\mathrm{X}$ & $\mathrm{X}$ & & \\
\hline Aulacoseira agassizii (Ostenf.) Simonsen & & $X$ & $\mathrm{X}$ & \\
\hline
\end{tabular}


Tabela 5 (continuação)

\begin{tabular}{|c|c|c|c|c|}
\hline Família / Espécie & JB & VB & LR & $\mathrm{BB}$ \\
\hline Aulacoseira agassizii (Ostenf.) Simonsen var. malayensis (Hust.) Simonsen & & $\mathrm{X}$ & $\mathrm{X}$ & \\
\hline Aulacoseira granulata (Ehr.) Simonsen var. granulata & $\mathrm{X}$ & $\mathrm{X}$ & $\mathrm{X}$ & \\
\hline Aulacoseira granulata (Ehr.) Simonsen var. angustissima (Mull.) Simonsen & $\mathrm{X}$ & $\mathrm{X}$ & $\mathrm{X}$ & \\
\hline Aulacoseira italica (Ehr.) Simonsen & $\mathrm{X}$ & $\mathrm{X}$ & $\mathrm{X}$ & \\
\hline Cyclotella meneghiniana Kütz. & $\mathrm{X}$ & $\mathrm{X}$ & $\mathrm{X}$ & $\mathrm{X}$ \\
\hline Discostella stelligera (Clev. \& Grun.) Houk \& Klee & $\mathrm{X}$ & $\mathrm{X}$ & & \\
\hline Gomphonema gracile Ehr. emend. V. Heurck & $\mathrm{X}$ & & & \\
\hline Melosira varians Agardh & $\mathrm{X}$ & & $\mathrm{X}$ & \\
\hline Pinnularia doeringhii Freng. & & $\mathrm{X}$ & $\mathrm{X}$ & \\
\hline Ulnaria ulna (Nitz.) Comp. & $\mathrm{X}$ & $\mathrm{X}$ & $\mathrm{X}$ & $\mathrm{X}$ \\
\hline \multicolumn{5}{|l|}{ CRYPTOPHYCEAE } \\
\hline Chroomonas nordstedtii Hansg. & & & & $\mathrm{X}$ \\
\hline Cryptomonas erosa Ehr. & $\mathrm{X}$ & $\mathrm{X}$ & $\mathrm{X}$ & $\mathrm{X}$ \\
\hline Cryptomonas marsonii Skuja & $\mathrm{X}$ & $\mathrm{X}$ & $\mathrm{X}$ & $\mathrm{X}$ \\
\hline Cryptomonas obovata Skuja & $\mathrm{X}$ & $\mathrm{X}$ & $\mathrm{X}$ & $\mathrm{X}$ \\
\hline Cryptomonas pyrenoidifera Geitler & $\mathrm{X}$ & $\mathrm{X}$ & $\mathrm{X}$ & $\mathrm{X}$ \\
\hline Cryptomonas tenuis Pasch. & $\mathrm{X}$ & & & \\
\hline Rhodomonas minuta Skuja var. nanoplanctonica Skuja & & & & $\mathrm{X}$ \\
\hline \multicolumn{5}{|l|}{ DINOPHYCEAE } \\
\hline Peridiniopsis thompsonii (Thomp.) Bourr. & $\mathrm{X}$ & $\mathrm{X}$ & $\mathrm{X}$ & $\mathrm{X}$ \\
\hline \multicolumn{5}{|l|}{ Peridinium gatunense Nyg. } \\
\hline Peridinium umbonatum Stein & $\mathrm{X}$ & & $\mathrm{X}$ & $\mathrm{X}$ \\
\hline Peridinium willei Huitf.-Kaas & $\mathrm{X}$ & & $\mathrm{X}$ & \\
\hline \multicolumn{5}{|l|}{ EUGLENOPHYCEAE } \\
\hline Lepocinclis cf. salina Fritsch & $\mathrm{X}$ & & & \\
\hline Lepocinclis fusiformis (Carter) Lemm. & & & $\mathrm{X}$ & \\
\hline Phacus agilis Skuja & $\mathrm{X}$ & & $\mathrm{X}$ & \\
\hline Phacus cf. triqueter (Ehr.) Duj. & $\mathrm{X}$ & $\mathrm{X}$ & $\mathrm{X}$ & $\mathrm{X}$ \\
\hline Phacus suecicus Lemm. & $\mathrm{X}$ & & & \\
\hline Trachelomonas volvocina $\mathrm{Ehr}$ & $\mathrm{X}$ & $\mathrm{X}$ & & $\mathrm{X}$ \\
\hline Trachelomonas volvocinopsis Swir. & $\mathrm{X}$ & $\mathrm{X}$ & & $\mathrm{X}$ \\
\hline \multicolumn{5}{|l|}{ CHLOROPHYCEAE } \\
\hline Actinastrum hantzschii Lager. & & $\mathrm{X}$ & & $\mathrm{X}$ \\
\hline Amphikrikos hexacosta (Thomps.) Hind. & $\mathrm{X}$ & $\mathrm{X}$ & $\mathrm{X}$ & $\mathrm{X}$ \\
\hline Ankistrodesmus bernardii Kom. & $\mathrm{X}$ & $\mathrm{X}$ & & \\
\hline Ankistrodesmus densus Kors. & $\mathrm{X}$ & $\mathrm{X}$ & & \\
\hline Ankistrodesmus fasciculatus (Lund.) Kom.-Legn. & & $\mathrm{X}$ & & \\
\hline Ankistrodesmus fusiformis Corda & $\mathrm{X}$ & $\mathrm{X}$ & $\mathrm{X}$ & \\
\hline Ankistrodesmus tortus Hind. & $\mathrm{X}$ & $\mathrm{X}$ & & \\
\hline Asteracys cubensis Comas & $\mathrm{X}$ & & $\mathrm{X}$ & $\mathrm{X}$ \\
\hline Asterococcus limneticus G.S. Smith & $\mathrm{X}$ & $\mathrm{X}$ & $\mathrm{X}$ & $\mathrm{X}$ \\
\hline Botryococcus protuberans West \& G.S. Smith & & & $\mathrm{X}$ & \\
\hline Botryococcus terribiles Kom. \& Marvan & & $\mathrm{X}$ & $\mathrm{X}$ & $\mathrm{X}$ \\
\hline Chlorella homosphaera Skuja & $\mathrm{X}$ & $\mathrm{X}$ & & $\mathrm{X}$ \\
\hline Chlorella oocystoides Hind. & & & & $\mathrm{X}$ \\
\hline Chlorella vulgaris Beij. & & & & $\mathrm{X}$ \\
\hline Chlorotetraedron incus (Teil.) Kom. \& Kov. & & & $\mathrm{X}$ & $\mathrm{X}$ \\
\hline Choricystis chodati (Jaag) Fott & & & $\mathrm{X}$ & $\mathrm{X}$ \\
\hline Choricystis minor (Skuja) Fott & $\mathrm{X}$ & $\mathrm{X}$ & $\mathrm{X}$ & $\mathrm{X}$ \\
\hline Coccomyxa lacustris (Chodat) Pasch. & & & & $\mathrm{X}$ \\
\hline Coelastrum astroideum De Not. & & & $\mathrm{X}$ & \\
\hline
\end{tabular}


Tabela 5 (continuação)

\begin{tabular}{|c|c|c|c|c|}
\hline Família / Espécie & JB & VB & LR & $\mathrm{BB}$ \\
\hline Coelastrum cambricum Arch. var. cristata Kamm. & & & $\mathrm{X}$ & \\
\hline Coelastrum polychordum (Kors.) Hind. & $X$ & $\mathrm{X}$ & $\mathrm{X}$ & $\mathrm{X}$ \\
\hline Coelastrum proboscideum Bohl. & $\mathrm{X}$ & $\mathrm{X}$ & $\mathrm{X}$ & $\mathrm{X}$ \\
\hline Coelastrum pseudomicroporum Kors. & $\mathrm{X}$ & & $\mathrm{X}$ & $\mathrm{X}$ \\
\hline Coelastrum pulchrum Schmidle & $\mathrm{X}$ & $\mathrm{X}$ & $\mathrm{X}$ & $\mathrm{X}$ \\
\hline Coelastrum reticulatum (Dang.) Senn & $\mathrm{X}$ & $\mathrm{X}$ & $X$ & $\mathrm{X}$ \\
\hline Coenochloris pelagica (Teil) Fott & & $\mathrm{X}$ & & \\
\hline Coenochloris piscinalis Fott & & & $\mathrm{X}$ & $\mathrm{X}$ \\
\hline Crucigenia tetrapedia (Kirch.) West \& G.S. West & & & $\mathrm{X}$ & \\
\hline Crucigeniella apiculata (Lemm.) Kom. & & & $\mathrm{X}$ & $\mathrm{X}$ \\
\hline Crucigeniella rectangularis (Näg.) Kom. & $\mathrm{X}$ & $\mathrm{X}$ & & $\mathrm{X}$ \\
\hline Desmodesmus armatus (Chodat) Hegew. & $\mathrm{X}$ & & $\mathrm{X}$ & $\mathrm{X}$ \\
\hline Desmodesmus armatus (Chodat) Hegew. var. bicaudatus (Gugl.) Hegew. & $\mathrm{X}$ & $\mathrm{X}$ & $\mathrm{X}$ & $\mathrm{X}$ \\
\hline Desmodesmus armatus (Chodat) Hegew. var. spinosus (Fritsch \& Rich) Hegew. & & & $\mathrm{X}$ & $\mathrm{X}$ \\
\hline Desmodesmus brasiliensis (Bohl.) Hegew. & & $\mathrm{X}$ & $\mathrm{X}$ & $\mathrm{X}$ \\
\hline Desmodesmus denticulatus (Lagerh.) An, Friedl \& Hegew. & & $\mathrm{X}$ & & $\mathrm{X}$ \\
\hline Desmodesmus hystrix (Lagerh.) Hegew. & & $\mathrm{X}$ & & $\mathrm{X}$ \\
\hline Desmodesmus intermedius (Chodat) Hegew. & $\mathrm{X}$ & $\mathrm{X}$ & $\mathrm{X}$ & $\mathrm{X}$ \\
\hline Desmodesmus lefevrei var. muzzanensis (Hub.-Pest.) Friedl \& Hegew. & $\mathrm{X}$ & $\mathrm{X}$ & & $\mathrm{X}$ \\
\hline Desmodesmus maximus (West \& G.S. West) Hegew. & $\mathrm{X}$ & $\mathrm{X}$ & $\mathrm{X}$ & $\mathrm{X}$ \\
\hline Desmodesmus opoliensis (Richt.) Hegew. var. alatus (Dedus.) Hegew. & $\mathrm{X}$ & & $\mathrm{X}$ & $\mathrm{X}$ \\
\hline Desmodesmus opoliensis (Richt.) Hegew. var. carinatus (Lemm.) Hegew. & $\mathrm{X}$ & & $\mathrm{X}$ & $\mathrm{X}$ \\
\hline Desmodesmus pannonicus (Hortob.) Hegew. & $\mathrm{X}$ & $\mathrm{X}$ & & $\mathrm{X}$ \\
\hline Desmodesmus perforatus (Lemm.) Hegew. & & & & $\mathrm{X}$ \\
\hline Desmodesmus protuberans (Fritsch \& Rich) Hegew. & & $\mathrm{X}$ & $\mathrm{X}$ & $\mathrm{X}$ \\
\hline Desmodesmus spinosus (Chodat) Hegew. & $\mathrm{X}$ & $\mathrm{X}$ & $\mathrm{X}$ & $\mathrm{X}$ \\
\hline Dicloster acuatus Jao & & $\mathrm{X}$ & $\mathrm{X}$ & $\mathrm{X}$ \\
\hline Dictyosphaerium elegans Bach. & $\mathrm{X}$ & & & \\
\hline Dictyosphaerium pulchellum Wood & $\mathrm{X}$ & $\mathrm{X}$ & $\mathrm{X}$ & $\mathrm{X}$ \\
\hline Dictyosphaerium tetrachotomum Printz. & $\mathrm{X}$ & $\mathrm{X}$ & $\mathrm{X}$ & $\mathrm{X}$ \\
\hline Didymocystis comasii Kom. & $\mathrm{X}$ & & & \\
\hline Dispora speciosa Kors. & & $\mathrm{X}$ & $\mathrm{X}$ & $\mathrm{X}$ \\
\hline Echinosphaeridium nordstedtii Lemm. & $\mathrm{X}$ & $\mathrm{X}$ & & \\
\hline Elakatothrix biplex (Nyg.) Hind. & & $\mathrm{X}$ & & \\
\hline Elakatothrix genevensis Hind. & $\mathrm{X}$ & & $\mathrm{X}$ & $\mathrm{X}$ \\
\hline Elakatothrix linearis Pasch. & $\mathrm{X}$ & & $\mathrm{X}$ & $\mathrm{X}$ \\
\hline Eutetramorus fottii (Hind.) Kom. & & $\mathrm{X}$ & $\mathrm{X}$ & $\mathrm{X}$ \\
\hline Eutetramorus planctonicus (Kors.) Bourr. & $\mathrm{X}$ & $\mathrm{X}$ & $\mathrm{X}$ & $\mathrm{X}$ \\
\hline Eutetramorus tetrasporus Kom. & & $\mathrm{X}$ & $\mathrm{X}$ & \\
\hline Fusola viridis Snow & $\mathrm{X}$ & & $\mathrm{X}$ & $\mathrm{X}$ \\
\hline Golenkinia paucispina West \& G. S. West & $\mathrm{X}$ & $\mathrm{X}$ & $\mathrm{X}$ & \\
\hline Golenkinia radiata Chodat & $\mathrm{X}$ & $\mathrm{X}$ & $\mathrm{X}$ & $\mathrm{X}$ \\
\hline Granulocystopsis coronata (Lemm.) Hind. var. elegans (Fott) Kom. & & & $\mathrm{X}$ & $\mathrm{X}$ \\
\hline Granulocystopsis pseudo-coronata (Kors.) Hind. & & & $\mathrm{X}$ & \\
\hline Kirchneriella aperta Teil. & & & $\mathrm{X}$ & \\
\hline Kirchneriella diane (Bohl.) Comas & $\mathrm{X}$ & $\mathrm{X}$ & $\mathrm{X}$ & $\mathrm{X}$ \\
\hline Kirchneriella incurvata Belch. \& Swale & & $\mathrm{X}$ & & \\
\hline Kirchneriella irregularis (Smith) Kors. & & & $\mathrm{X}$ & \\
\hline Kirchneriella lunaris (Kirch.) Moeb. & $\mathrm{X}$ & & & $\mathrm{X}$ \\
\hline Kirchneriella obesa (West) Schmidle & $\mathrm{X}$ & $\mathrm{X}$ & $\mathrm{X}$ & $\mathrm{X}$ \\
\hline Kirchneriella rotunda (Kors.) Hind. & & $\mathrm{X}$ & & \\
\hline
\end{tabular}


Tabela 5 (continuação)

\begin{tabular}{|c|c|c|c|c|}
\hline Família / Espécie & JB & VB & LR & BB \\
\hline Koliella longiseta (Visch.) Hind. & $\mathrm{X}$ & $\mathrm{X}$ & & $\mathrm{X}$ \\
\hline Koliella cf. spiralis Kuosa & $\mathrm{X}$ & & & \\
\hline Lobocystis planctonica (Tiff. \& Ahlstr) Fott & & & $\mathrm{X}$ & \\
\hline Micractinium pusillum Fres. & $\mathrm{X}$ & & $\mathrm{X}$ & $\mathrm{X}$ \\
\hline Monoraphidium arcuatum (Kors.) Hind. & $\mathrm{X}$ & $\mathrm{X}$ & $\mathrm{X}$ & $\mathrm{X}$ \\
\hline Monoraphidium circinale (Nyg.) Nyg. & $\mathrm{X}$ & $\mathrm{X}$ & $\mathrm{X}$ & $\mathrm{X}$ \\
\hline Monoraphidium contortum (Thur.) Kom.-Legn. & $\mathrm{X}$ & $\mathrm{X}$ & $\mathrm{X}$ & $\mathrm{X}$ \\
\hline \multicolumn{5}{|l|}{ Monoraphidium convolutum (Corda) Kom.-Legn. } \\
\hline Monoraphidium griffithii (Berk.) Kom.-Legn. & $\mathrm{X}$ & $\mathrm{X}$ & $\mathrm{X}$ & $\mathrm{X}$ \\
\hline \multicolumn{5}{|l|}{ Monoraphidium irregulare (Smith) Kom.-Legn. } \\
\hline Monoraphidium komarkovae Nyg. & $\mathrm{X}$ & $\mathrm{X}$ & $\mathrm{X}$ & $\mathrm{X}$ \\
\hline Monoraphidium longiusculum Hind. & $\mathrm{X}$ & $\mathrm{X}$ & $\mathrm{X}$ & $\mathrm{x}$ \\
\hline Monoraphidium minutum (Nag.) Kom.-Legn. & $\mathrm{X}$ & $\mathrm{X}$ & $\mathrm{X}$ & $\mathrm{x}$ \\
\hline Monoraphidium nanum (Ettl) Hind. & $\mathrm{X}$ & $\mathrm{X}$ & $\mathrm{X}$ & $\mathrm{X}$ \\
\hline Nephrochlamys danica Kom. & $\mathrm{X}$ & & $\mathrm{X}$ & \\
\hline Nephrochlamys willeana (Printz) Kors. & & & $X$ & \\
\hline Nephrocytium agardhianum Nag. & $\mathrm{X}$ & $\mathrm{X}$ & $\mathrm{X}$ & $\mathrm{X}$ \\
\hline Nephrocytium allanthoideum Bohl & $\mathrm{X}$ & & $\mathrm{X}$ & $\mathrm{x}$ \\
\hline Nephrocytium schilleri (Kamm.) Com. & & & $\mathrm{X}$ & $\mathrm{X}$ \\
\hline Oocystis borgei Snow & & $\mathrm{X}$ & $\mathrm{X}$ & $\mathrm{X}$ \\
\hline Oocystis lacustris Chodat & $\mathrm{X}$ & $\mathrm{X}$ & $\mathrm{X}$ & $\mathrm{x}$ \\
\hline Oocystis naegelli A. Br. & $\mathrm{X}$ & & $\mathrm{X}$ & $\mathrm{X}$ \\
\hline Oocystis tainoensis Kom. & $\mathrm{X}$ & & $\mathrm{X}$ & \\
\hline Pediastrum angulosus (Ehr.) Menegh. & $\mathrm{X}$ & & & \\
\hline Pediastrum biradiatum Meyen & $\mathrm{X}$ & & $X$ & \\
\hline Pediastrum duplex Meyen & $\mathrm{X}$ & $\mathrm{X}$ & $\mathrm{X}$ & $\mathrm{X}$ \\
\hline Pediastrum simplex Meyen & $\mathrm{X}$ & $\mathrm{X}$ & $\mathrm{X}$ & $\mathrm{X}$ \\
\hline Pediastrum tetras (Ehr.) Ralfs & $\mathrm{X}$ & $\mathrm{X}$ & $\mathrm{X}$ & \\
\hline Pseudodidymocystis fina (Kom). Hegew \& Deason & $\mathrm{X}$ & & & $\mathrm{X}$ \\
\hline Pseudokirchneriella danubiana (Hind.) Hind. & $\mathrm{X}$ & $\mathrm{X}$ & $\mathrm{X}$ & $\mathrm{X}$ \\
\hline Quadricoccus leave Fott & $\mathrm{X}$ & & & \\
\hline Radiococcus nimbatus (De Wild) Schmidle & $\mathrm{X}$ & & $\mathrm{X}$ & $\mathrm{X}$ \\
\hline Radiococcus planktonicus Lund & $\mathrm{X}$ & $\mathrm{X}$ & & $\mathrm{X}$ \\
\hline Raphidiocelis contorta (Schmidle) Marvan, Kom. \& Comas & $\mathrm{X}$ & $\mathrm{X}$ & & $\mathrm{x}$ \\
\hline Raphidiocelis subcaptata (Kors.) Marvan, Kom. \& Comas & $\mathrm{X}$ & $\mathrm{X}$ & $\mathrm{X}$ & $\mathrm{X}$ \\
\hline Scenedesmus acuminatus (Lagerh) Chodat & $\mathrm{X}$ & & $\mathrm{X}$ & $\mathrm{X}$ \\
\hline Scenedesmus arcuatus Lemm. & $\mathrm{X}$ & & $\mathrm{X}$ & $\mathrm{X}$ \\
\hline Scenedesmus bacillaris Gutw. & $\mathrm{X}$ & & & \\
\hline Scenedesmus bijugus (Turpin) Kutz. & & & & $\mathrm{X}$ \\
\hline Scenedesmus disciformis (Chodat) Fott \& Kom. & & $\mathrm{X}$ & $\mathrm{X}$ & $\mathrm{X}$ \\
\hline Scenedesmus ellipticus Corda & $\mathrm{X}$ & $\mathrm{X}$ & $\mathrm{X}$ & $\mathrm{X}$ \\
\hline Scenedesmus indicus Phil. & $\mathrm{X}$ & & $\mathrm{X}$ & $\mathrm{x}$ \\
\hline Scenedesmus javanensis Chodat & $\mathrm{X}$ & $\mathrm{X}$ & $\mathrm{X}$ & $\mathrm{X}$ \\
\hline Scenedesmus magnus Meyen & $\mathrm{X}$ & $\mathrm{X}$ & $\mathrm{X}$ & $\mathrm{X}$ \\
\hline Scenedesmus obliquus (Turp.) Kütz. & $\mathrm{X}$ & & $\mathrm{X}$ & $\mathrm{X}$ \\
\hline Scenedesmus opoliensis Richt. var. danubialis Hortob. & & $\mathrm{X}$ & $\mathrm{X}$ & \\
\hline Scenedesmus opoliensis Richt. var. mononensis Chodat & $\mathrm{X}$ & $\mathrm{x}$ & $\mathrm{X}$ & $\mathrm{X}$ \\
\hline Scenedesmus ovalternus Chodat var. graevenitzii (Ber.) Chodat & & $\mathrm{X}$ & & $\mathrm{X}$ \\
\hline Scenedesmus regularis Svir. & & & $\mathrm{X}$ & $\mathrm{X}$ \\
\hline Schroederia spiralis (Printz) Kors. & $\mathrm{X}$ & & & $\mathrm{X}$ \\
\hline Selenastrum bibraianum Rein. & $\mathrm{X}$ & & & \\
\hline
\end{tabular}


Tabela 5 (continuação)

\begin{tabular}{|c|c|c|c|c|}
\hline Família / Espécie & JB & VB & LR & $\mathrm{BB}$ \\
\hline Selenastrum gracile Rein. & $\mathrm{X}$ & $\mathrm{X}$ & $\mathrm{X}$ & $\mathrm{X}$ \\
\hline Selenastrum rinoi Kom. \& Comas & $\mathrm{X}$ & $\mathrm{X}$ & & $\mathrm{X}$ \\
\hline Sphaerellopsis fluviatilis (Stein) Pasch & $\mathrm{X}$ & $\mathrm{X}$ & $\mathrm{X}$ & \\
\hline Tetrachlorella alternans (G.M. Smith) Kors. & $\mathrm{X}$ & & & \\
\hline Tetradesmus wisconsinensis G.M. Smith & & $\mathrm{X}$ & & \\
\hline Tetraedron caudatum (Corda) Hansg. & $\mathrm{X}$ & $\mathrm{X}$ & $\mathrm{X}$ & \\
\hline Tetraedron minimum (A. Br.) Hansg. & $\mathrm{X}$ & $\mathrm{X}$ & $\mathrm{X}$ & $\mathrm{X}$ \\
\hline Tetraedron triangulare Kors. & $\mathrm{X}$ & & $\mathrm{X}$ & \\
\hline Tetrallantos lagerheimii Teil. & $\mathrm{X}$ & $\mathrm{X}$ & $\mathrm{X}$ & $\mathrm{X}$ \\
\hline Tetranephris brasiliensis Leit. \& Bic. & $\mathrm{X}$ & $\mathrm{X}$ & $\mathrm{X}$ & $\mathrm{X}$ \\
\hline Tetrastrum glabrum (Roll) Ahlstr. \& Tiff. & $\mathrm{X}$ & & & \\
\hline Tetrastrum komarekii Hind. & $\mathrm{X}$ & $\mathrm{X}$ & $\mathrm{X}$ & $\mathrm{X}$ \\
\hline \multicolumn{5}{|l|}{ Tetrastrum staurogeniaeforme (Schröd.) Lemm. } \\
\hline Tetrastrum triangulare (Chodat) Kom. & $\mathrm{X}$ & $\mathrm{X}$ & $\mathrm{X}$ & $\mathrm{X}$ \\
\hline Thorakochloris planctonica Fott & $\mathrm{X}$ & $\mathrm{X}$ & $\mathrm{X}$ & $\mathrm{X}$ \\
\hline Treubaria schmidlei (Schmidle) Fott \& Kov. & & & $\mathrm{X}$ & \\
\hline Westella botryoides (West) De Wild. & $\mathrm{X}$ & $\mathrm{X}$ & $\mathrm{X}$ & $\mathrm{X}$ \\
\hline \multicolumn{5}{|l|}{ ZYGNEMAPHYCEAE } \\
\hline Cosmarium contractum Kirchner var. minutum (Delp.) West \& G.S.West & $\mathrm{X}$ & & $\mathrm{X}$ & $\mathrm{X}$ \\
\hline Staurastrum laeve Ralfs & $\mathrm{X}$ & & $\mathrm{X}$ & $\mathrm{X}$ \\
\hline Staurastrum tetracerum (Kutz.) Ralfs var. tortum (Teil.) Borge & $\mathrm{X}$ & $\mathrm{X}$ & $\mathrm{X}$ & $\mathrm{X}$ \\
\hline Staurastrum volans West \& G.S. West & $\mathrm{X}$ & $\mathrm{X}$ & $\mathrm{X}$ & $\mathrm{X}$ \\
\hline Staurodesmus triangularis (Lagerh.) Teil. & $\mathrm{X}$ & & $\mathrm{X}$ & $\mathrm{X}$ \\
\hline
\end{tabular}

Considerando o índice de similaridade de Jaccard (figura 5), observou-se a formação de dois agrupamentos, um do Lago do Jardim Botânico e Lago do Parque Vaca Brava que tiveram uma similaridade na composição do fitoplâncton, enquanto no Lago do Bosque dos Buritis e Lago das Rosas apresentaram um fitoplâncton mais similar. No entanto, os coeficientes de similaridade apresentados por esses lagos variaram de 0,45 a 0,52 , indicando uma baixa similaridade florística, sugerindo, que mesmo tendo ocorrido esse agrupamento, a composição fitoplanctônica entre os lagos é bastante diferente, reforçando a heterogeneidade nos lagos registrada pela diversidade beta.

Os lagos artificiais urbanos estudados são rasos e analisando as características morfométricas (tabela 1) e paisagística de cada lago, o Lago do Jardim Botânico e o Lago do Parque Vaca Brava foram semelhantes em características, tais como margem não cimentada, forma do lago alongada e o contínuo aporte de água das nascentes. O Lago das Rosas e o Lago do Bosque dos Buritis assemelharam-se por apresentarem margem cimentada, forte impacto de visitação pública e águas com problemas em suas nascentes (o primeiro recebe o esgoto do Jardim

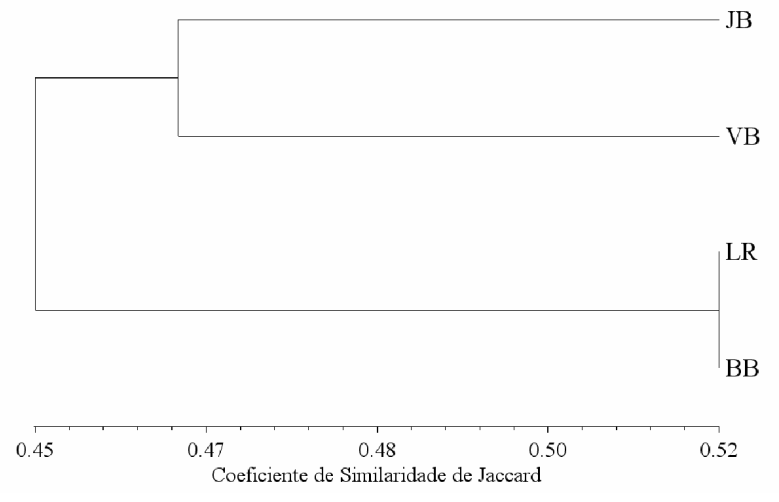

Figura 5. Dendrograma do coeficiente de similaridade de Jaccard, obtidos com base nos inventários florísticos de cada lago $(r=0,72)$. Lagos: (JB) Lago do Jardim Botânico; (VB) Lago Vaca Brava; (LR) Lago das Rosas e (BB) Lago do Bosque dos Buritis.

Zoológico de Goiânia e o segundo teve o aporte de água das nascentes interrompido durante o período de estudo). No entanto, limnologicamente, o Lago do Bosque dos Buritis apresentou-se diferente, na maior parte do estudo, dos demais lagos, da mesma forma com o Lago do Jardim Botânico, enquanto que o Lago 
Tabela 6. Valores de riqueza de espécies do fitoplâncton observada (diversidade gama) e riqueza estimada.

\begin{tabular}{lcccc}
\hline & Diversidade gama & Sjack1 & Sjack2 & Schao2 \\
\hline Lago do Jardim Botânico & 194 & 275 & 316 & 294 \\
Lago Vaca Brava & 171 & 251 & 291 & 265 \\
Lago das Rosas & 211 & 292 & 329 & 297 \\
Lago do Bosque dos Buritis & 179 & 261 & 314 & 337 \\
\hline
\end{tabular}

do Parque Vaca Brava e do Lago das Rosas foram mais semelhantes.

Os elevados valores de diversidade beta e baixos valores de similaridade ocorreram devido à heterogeneidade existente entre os lagos e a tendência a sazonalidade, influenciada pela precipitação (meses de seca e chuva). As diferentes características ambientais e morfométricas dos lagos proporcionaram uma composição fitoplanctônica distinta. Apesar dos quatro lagos serem urbanos, alguns receberam maior visita da população (Lago do Parque Vaca Brava, Lago das Rosas e o Lago do Bosque dos Buritis), apresentaram características paisagísticas diferentes, como chafariz (Lago do Bosque dos Buritis), veículos aquáticos (pedalinho) que permitem passeios no lago (Lago das Rosas e Lago do Parque Vaca Brava) e área de repouso para pássaros migrantes, juntamente com criação de marrecos e patos (Bosque dos Buritis). Todas essas características proporcionaram uma diferenciação limnológica nos lagos e, por conseqüência, considerouse que puderam afetar a composição do fitoplâncton em especial pelos resíduos de dejetos orgânicos que forneceram nutrientes aos lagos.

As políticas de conservação são baseadas principalmente em riqueza de espécies. Segundo Tocker et al. (1999) a variabilidade ambiental e as mudanças na composição biológica (beta diversidade) devem ser levadas em conta para o desenvolvimento de estratégias para a conservação. Para Bridgewater $e t$ al. (2004) um conhecimento completo da diversidade beta é vital para a seleção de áreas para a conservação e um estudo cuidadoso da composição biológica é essencial para assegurar a seleção de reservas adequadas para proteção do ecossistema. No entanto, pouco se sabe sobre os indicativos para preservação de ecossistemas aquáticos. Nesse sentido, observouse que a diversidade beta poderá vir a ser mais uma ferramenta para indicar áreas prioritárias para medidas de conservação na área do presente estudo.

Os lagos estudados neste trabalho têm uma importância ambiental e paisagística para a população local. No entanto, esses lagos vêm sofrendo impactos ambientais, provocando incômodos e preocupações à população. Florações de Chlorophyceae e Cyanobacteria provocam odores que frequientemente afetam aos visitantes e moradores do entorno dos parques, associados à diminuição da qualidade cênica. Acredita-se que um monitoramento deva ser realizado para o planejamento de políticas conservacionistas. Através dos resultados obtidos neste trabalho pode-se inferir o delineamento de políticas conservacionistas. Como descrito anteriormente, o lago do Jardim Botânico e o lago do Parque Vaca Brava apresentam características morfométricas semelhantes e o lago do Bosque dos Buritis e o lago das Rosas também. Essas características favoreceram uma composição fitoplanctônica semelhante. Assim, um programa de manejo para a conservação deve ser efetuado de forma semelhante para os lagos do Jardim Botânicos e do Parque Vaca Brava e também um manejo que atenda o lago do Bosque dos Buritis e lago das Rosas.

Os lagos estudados são rasos e apresentaram características limnológicas distintas, evidenciando a influência das características morfométricas de cada lago sobre as variáveis físicas e químicas.

De forma geral, a riqueza específica total (diversidade gama) foi elevada nos lagos pelo tipo de amostragem realizada e os extrapoladores utilizados, permitindo concluir que a amostragem nos lagos foi bem expressiva. Os lagos registraram elevados valores de diversidade beta influenciados pela sazonalidade e as características morfométricas únicas de cada lago.

Os ambientes com composição florística mais semelhante foram os lagos do Jardim Botânico e Parque Vaca Brava e os lagos do Bosque dos Buritis e das Rosas. O lago das Rosas apresentou maior riqueza específica nos meses chuva, enquanto no Lago do Bosque dos Buritis ocorreu nos meses de seca. Apesar de lagos urbanos e eutrofizados a biodiversidade foi elevada.

No presente estudo, o fitoplâncton dos lagos artificiais urbanos foi caracterizado principalmente 
pela elevada riqueza de espécies, destacando-se Chlorophyceae como a classe com maior número de táxons. Os lagos estudados apresentaram composição específica distinta, portanto diferenciados como indicado pela diversidade beta.

\section{Agradecimentos}

Agradecemos a Agência Ambiental do Estado de Goiás pelo auxílio nos trabalhos de campo, e disponibilidade de uso do laboratório de físico-químico. A Dra. Maria Helena Rezende (Botânica-Universidade Federal de Goiás) pelo uso do microscópio para análise dos táxons. A Dra. Mariângela Menezes (Museu Nacional/UFRJ) pela confirmação da identificação dos fitoflagelados.

\section{Literatura citada}

APHA. 1995. American Public Heath Association. Standard methods for the examination of water and wastewater. 19 ed. APHA-AWWA-WPCF, Washington D.C.

Bicudo, C. E. M. \& Menezes, M. 2006. Gêneros de algas de águas continentais do Brasil: chave para identificação e descrições. 2 ed. RiMa Editora, São Carlos.

Bini, L.M., Thomaz, S.M. \& Souza, D.C. 2001. Species richness and $\beta$-diversity of aquatic macrophytes in the Upper Paraná River floodplain. Archives für Hydrobiologie 151: 511-525.

Branco, C.W.C. \& Senna, P.A.C. 1996. Phytoplankton composition, community structure and seasonal changes in a tropical reservoir (Paranoá Reservoir, Brazil). Algological Studies 81:69-84.

Bridgewater, S., Ratter, J.A. \& Ribeiro, J.F. 2004. Biogeographic patterns, $\beta$-diversity and dominance in the cerrado biome of Brazil. Biodiversity and Conservation 13: 2295-2318.

Burnham, K.P. \& Overton, W.S. 1978. Estimation of the size of a closed population when capture probabilities vary among animals. Biometrika 65: 625-633

Chao, A.1987. Estimating the population size for capturerecapture data with unequal catchability. Biometrics 43: 783-791.

Chazdon, R.L., Colwell, R.K., Denslow, J.S. \& Guariguata, M.R. 1998. Statistical methods for estimating species richness of woody regeneration in primary and secondary rain forests of Northeastern Costa Rica. In: F. Dallmeier \& J. A. Comiskey (eds.). Forest biodiversity research, monitoring and modeling. The Parthenon Publishing Group, Washington, pp. 285-309.

Clesceri, L.S., Greenberg, A.E. \& Trussell, R.R. 1992. Métodos normalizados para el analisis de aguas potables y residuales. Ediciones Diaz de Santos S.A/American Public Health Association, American Water Works Association/ Water Pollution Control Federation, Madrid.

Colwell, R. K. 1997. Estimates 5: Statistical Estimation of Species Richness and Shared Species from Samples. User Guide, 22p. http://www.viceroy.eeb.uconn.edu/ estimates. (acesso em 16.05.2006).

Comas, A. 1996. Las Chlorococcales dulciacuicolas de Cuba. Bibliotheca Phycologica, Band 99. J.Cramer, Stuttgart.

Ettl, H. 1983. Chlorophyta I. In: H. Ettl, J. Gerloff, H. Heynig \& D. Mollenhauer (eds.). Süsswasserflora von Mitteleuropa, Band 9. Gustav Fischer Verlag, Stuttgart

Ferreira, A.C.S. \& Menezes, M. 2000. Flora planctônica de um reservatório eutrófico, lagoa Guandu, município de Nova Iguaçu, RJ. Hoehnea 27: 45-76.

Galinkin, M. 2002. Geogoiás. ed. Goiânia: Agência Ambiental de Goiás: Fundação CEBRAC / PNUMA / SEMARH, Goiânia.

Gaston, K.J. 1996. Biodiversity. A biology of number of difference. Blackwell, Oxford.

Giani A., Figueiredo C.C. \& Eterovick P.C. 1999. Algas planctônicas do reservatório da Pampulha (MG): Euglenophyta, Chrysophyta, Pyrrophyta, Cyanobacteria. Revista Brasileira de Botânica 22:107-116.

Harrison, S., Ross, S.J. \& Lawton, J.H. 1992. Beta diversity on geographic gradients in Britain. Journal of Animal Ecology 62: 151-158.

Hindák, F. 1990. Studies on the chlorococcal algae (Chlorophyceae ) V. Biolgické Práce 36 : 1-225.

Huszar, V.L.M., Silva, L.H.S. \& Esteves, F.A. 1990. Estrutura das comunidades fitoplanctônicas de 18 lagoas da região do Baixo Rio Doce, Linhares, Espírito Santo, Brasil. Revista Brasileira de Biologia 50: 585-598.

Jaccard, P. 1908. Nouvelles recherches sur la distribution florale. Bulletin de la Societé Vanddoise des Sciences Natureles 44: 223-270.

Komárek, J. \& Anagnostidis, K. 1989. Modern aprroach to the classification system of cyanophytes, 4 : Nostocales. Algological Studies 56: 247-345.

Komárek, J. \& Anagnostidis, K. 1999. Cyanoprokaryota I. Teil Chroococcales. In: H. Ettl, G. Gärtner, H. Heynig \& D. Mollenhauer (eds.). Süsswasserflora von Mitteleuropa 19. G. Fischer, Verlag, pp. 1-548.

Komárek, J. \& Fott, B. 1983. Chlorophyceae (Grünalgen), Ordiniung: Chlorococcales. In: G. Huber-Pestalozzi, H. Heynig \& D. Mollenhauer (eds.). Das Phytoplankton des Sübwassers: Systematik und Biologie. E. Schwiezerbat'sche Verlagsbuchlandlung, Stuttgart, pp. 1-1.044.

Krammer, K. \& Lange-Bertalot, H. 1991. Bacillariophyceae: Centrales, Fragilariaceae, 
Eunotiaceae. In: H. Ettl, J. Gerloff, H. Heynig \& D. Mollenhauer (eds.). Süsswasser Flora von Mittleleuropa. Band 2/4. Gustav Fischer Verlag, Stuttgart, pp. 1-576.

Lee, S.M. \& Chao, A. 1994. Estimating population size via sample coverage for closed capture-recapture models. Biometrics 50: 88-97.

Magurran, A.F. 2004. Measuring Biological diversity. Blackwell, Oxford.

Margalef, R. 1972. Homage to Evelyn Hutchinson, or why is there upper limit to diversity. Transactions of the Illinois State Academy of Science 44: 221-235.

Margalef, R. 1983. Ecologia. Omega, Barcelona.

McCune, B \& Mefford M.J. 1997. Multivariate analysis of ecological data Version 3.0. MjM Sotware, Gleneden Beach.

Melo, A.S. \& Froehlich, C.G. 2001. Macroinvertebrates in neotropical streams: richness patterns along a catchment and assemblage structure between 2 seasons. Journal of the North American Benthological Society 20: 1-16.

Melo, A.S., Pereira, R.A.S. , Santos, A.J. , Shepherd, G.J., Machado, G., Medeiros, H.F. \& Sawaya, R.J. 2003. Comparing species richness among assemblages using sample units: why not use extrapolation methods to compare species richness? Oikos 101: 398-410.

Menezes, M. 1999. Flora ficológica da Quinta da Boa Vista, RJ: taxonomia e estratégias de populações de Chlorophyceae flageladas em um lago artificial com déficit hídrico. Hoehnea 26: 107-120.

Nabout, J.C., Nogueira, I.S., Oliveira, L.G. \& Morais, R.R. 2007. Phytoplankton diversity (alpha, beta and gamma) from the Araguaia River tropical floodplain lakes (central Brazil) Hydrobiologia 575: 455-461.

Nogueira, I.S. \& Leandro-Rodrigues, N.C. 1999. Algas Planctonicas de um lago artifical do Jardim Botânico Chico Mendes, Município de Goiânia, Goiás. Revista Brasileira de Biologia 59: 377-395.

Prescott, G.W. \& Bicudo, C.E.M. 1981. A synopsis of North American desmids. 2. Desmidiaceae: Placodermae. Section 3. Lincoln \& London. University of Nebrasca Press, Lincoln.

Prescott, G.W., Bicudo, C.E.M. \& Vinyard, W.C. 1982. A synopsis of North Americam desmids. 2. Desmidiaceae: Placodermae. Section 4. Lincoln \& London. University of Nebraska Press, Lincoln.

Sant'Anna, C.L., Azevedo, M.T.P. \& Sormus, L. 1989. Fitoplâncton do lago das Garças, Parque Estadual das Fontes do Ipiranga, São Paulo, SP, Brasil: Estudos Taxonômicos e Aspectos Ecológicos. Hoehnea 16: 89-131.

Sant'Anna, C.L., Sormus, L., Tucci, A. \& Azevedo, M.T.P. 1997. Variação Sazonal do fitoplâncton do Lago das Garças, São Paulo, SP. Hoehnea 24: 67-86.
Scheffer, M. 1999. Ecology of shallow lakes. Chapman \& Hall, New York.

Shannon, C.E. \& Weaver, W. 1963. The mathematical theory of communication. Illinois University Press, Urbana.

Silva, L.S. 1999. Fitoplâncton de um reservatório eutrófico (lago Monte Alegre), Ribeirão Preto, São Paulo, Brasil. Revista Brasileira de Biologia 59: 281-303.

Starmach, K. 1985. Chrysophyceae and Haptophyceae. In: H. Ettl, J. Gerloff \& H. Heynig (eds.) Süsswasserflora von Mittleuropa, Band 1. Gustav Fischer Verlag, Stuttgart, pp. 1-515.Sneath, P.H. \& Sokal, R.R. 1973. Numerical taxonomy: The principles and practice of numerical classification. W. H. Freeman, San Francisco.

Talamoni, J.L.B. \& Okano W.Y. 1997. Limnological characterization and plankton community structure in aquatic systems of different trophic state. Verhandlungen der internationalen Vereinigung für Theorische und Angewandte Limnologie 26: 629-636.

Tockner, K., Schiemer F., Baumgartner C., Kum, G., Weigand, E., Zweimuller, I. \& Ward J.V. 1999. The Danube restoration project: Species diversity patterns across connectivity gradients in the floodplain system. Regulated Rivers: Research \& Management 15: 245 258.

Tucci A. \& Sant'Anna C.L. 2003. Cylindrospermopsis raciborskii (woloszynska) Seenayya \& Subba Raju (cyanobacteria): variação semanal e relações com fatores ambientais em um reservatório eutrófico, São Paulo, SP, Brasil. Revista Brasileira de Botânica 26: 97-112.

Tucci, A., Sant'Anna, C.L., Gentil, R.C. \& Azevedo, M.T.P. 2006. Fitoplâncton do Lago das Garças, São Paulo, Brasil: um reservatório urbano e eutrófico. Hoehnea 33: 147-175.

Utermöhl, H. 1958. Zur Vervollkommung der quantitativen phytoplankton-methodik. Internationale Vereinigung für Theoretiche und Angewandte Limnologie 9: 1-38.

Van den Hoeck, C., Mann, D.G. \& Jahns, H.M. 1993. Algae: an introduction to phycology. Cambridge University Press, Cambridge.

Vollenweider, R.A. 1974. A manual on methods for measuring primary production in aquatic environments. 2 ed. IBP, Handbook $n^{-0}$ 12. Blackwell Scientific Publications, London.

Ward, J.V., Tockner, K. \& Schiemer, F. 1999. Biodiversity of floodplain river ecosystems: ecotones and connectivity. Regulation River: Reservoir \& Management 11: 105-119.

Xiangcan, J. 2003. Analysis of eutrophication state and trend for lakes in China. Journal Limnological 62: 60-66. 